ABSTRACT: Reliable unipedal balance is fundamental to safe ambulation. Accordingly, older persons with peripheral neuropathy (PN), who are at increased risk for falls, demonstrate impaired unipedal balance. To explore the relationship between afferent function and unipedal balance, frontal plane proprioceptive thresholds at the ankle were quantified in 22 subjects (72.5 \pm 6.3 years; 11 with PN and 11 matched controls) while they were standing using a foot cradle system and a staircase series of 100 rotational stimuli. PN subjects, as compared to controls, demonstrated shorter median unipedal balance times $(3.4 \pm 2.7$ vs. $14.3 \pm 8.9 \mathrm{~s} ; P=0.0017)$ and greater (less precise) combined ankle inversion/eversion proprioceptive thresholds $\left(1.17 \pm 0.36\right.$ vs. $\left.0.65 \pm 0.37^{\circ} ; P=0.0055\right)$. Combined ankle inversion/ eversion proprioceptive thresholds explained approximately half the variance in unipedal balance time $\left(\mathrm{R}^{2}=0.5138 ; P=0.0004\right)$. Given prior work demonstrating a similarly strong relationship between ankle torque generation and unipedal balance, neuropathy-associated impairments in ankle frontal plane afferent and efferent function appear to be equally responsible for the inability of older persons with PN to reliably balance on one foot. They therefore provide distinct targets for intervention.

Muscle Nerve 39: 150-157, 2009

\title{
FRONTAL PLANE ANKLe PROPRIOCEPTIVE THRESHOLDS AND UNIPEDAL BALANCE
}

\author{
JAEBUM SON, PhD, ${ }^{1}$ JAMES A. ASHTON-MILLER, PhD, ${ }^{1}$ and JAMES K. RICHARDSON, MD ${ }^{2}$ \\ ${ }^{1}$ Department of Biomedical Engineering, Biomechanical Research Laboratory, University of \\ Michigan, 2350 Hayward St, 3216 G.G. Brown, Ann Arbor, Michigan 48109, USA \\ ${ }^{2}$ Department of Physical Medicine and Rehabilitation, University of Michigan Health System, \\ 325 E. Eisenhower Parkway, Ann Arbor, Michigan, 48108 USA
}

Accepted 9 September 2008

A diffuse peripheral neuropathy (PN) is a common neuromuscular disorder among older persons, with a prevalence of $\approx 30 \%$ in the 65 -84-year age group. ${ }^{20}$ Diabetes mellitus is the most common cause of PN, and the prevalence among those with this disorder increases with disease duration. PN affects about $10 \%$ of patients at the time of diagnosis, and the prevalence increases to about $50 \%$ after 5 years. $^{3}$ The disorder has functional significance, since it has been linked to a markedly increased risk of falls, ${ }^{25,26}$ particularly injurious falls. ${ }^{5}$ Older persons with PN have also shown diminished ability to achieve and maintain unipedal balance (UPB) as compared to older controls. ${ }^{24}$ Moreover, decreased UPB time has

\footnotetext{
Abbreviations: CMAP, compound muscle action potential; MDNS, Michigan Diabetes Neuropathy Score; PN, peripheral neuropathy; $\mathrm{TH}_{75}$, ankle proprioceptive threshold a subject is able to perceive with a $75 \%$ probability of success; $\mathrm{TH}_{75 \text { inv }}, \mathrm{TH}_{75}$ for ankle inversion; $\mathrm{TH}_{75 \mathrm{ev}}, \mathrm{TH}_{75}$ for ankle eversion; $\mathrm{TH}_{75 \text { inv }+ \text { ev }}$, the sum of the $\mathrm{TH}_{75}$ values for ankle inversion and eversion; UPB, unipedal balance

Key words: neuropathy; postural equilibrium; accidental falls; proprioception Correspondence to: J.K. Richardson; e-mail: jkrich@umich.edu
}

(C) 2009 Wiley Periodicals, Inc.

Published online 8 February 2009 in Wiley InterScience (www.interscience. wiley.com). DOI 10.1002/mus.21194 been identified as a marker for falls and injurious falls among older persons in general ${ }^{32}$ and those with PN. ${ }^{22}$ Therefore, an understanding of the specific biomechanical impairments that prevent patients with PN from achieving reliable unipedal balance is clinically relevant.

In previous work we found that distal lower extremity efferent function, more specifically ankle inversion rate of torque generation, explained slightly more than half the variance in clinical UPB in a group of diabetic older women with and without PN. ${ }^{12}$ We also found that older persons with PN showed impairment in $\mathrm{UPB}^{24}$ and increased frontal plane proprioceptive thresholds at the ankle as compared to older control subjects. ${ }^{31}$ However, the effect of frontal plane ankle proprioceptive thresholds on the ability to maintain UPB has not been explored; thus, the purpose of this study was to determine the relationship between UPB and frontal plane proprioceptive thresholds at the ankle. Proprioceptive thresholds in the frontal plane were explored, as opposed to those in the sagittal, because of the clinical observation that patients performing UPB lose their balance mediolaterally rather than antero- 
posterially. Research confirms the greater injury potential of lateral falls. ${ }^{11}$ The primary hypothesis was that increased frontal plane ankle proprioceptive thresholds would be inversely associated with UPB time. The secondary hypotheses were that subjects with PN would demonstrate increased ankle proprioceptive thresholds and decreased UPB times as compared to age and gender matched subjects without PN.

\section{MATERIALS AND METHODS}

Subjects. Twenty-two subjects, 11 with PN and 11 matched control subjects without PN, were recruited for the study. The PN subjects were recruited from the University of Michigan Electrodiagnostic Laboratory and the University of Michigan Orthotics and Prosthetics Center. The control subjects were recruited with the assistance of the Human Subjects Core within the University of Michigan Geriatric Center, which maintains a listing of older persons within the surrounding community who are willing to participate in research. All procedures were approved by the Institutional Review Board and all subjects provided written informed consent.

Inclusion criteria for all subjects were: 1) age 50-85 years; 2) ability to speak and understand English; 3) ankle dorsiflexion strength $\geq$ MRC grade 4 by manual muscle testing; and 4) ability to ambulate at least household distances without an assistive device. Subjects were excluded if they had abnormal vision despite correction; weight greater than $136 \mathrm{~kg}$ (300 pounds); evidence on physical examination of central nervous system dysfunction or musculoskeletal abnormality such as severe scoliosis or amputation.

All PN subjects had a known history of diabetes mellitus treated by diet, oral hypoglycemic, and/or insulin therapy. These subjects also all reported symptoms consistent with PN; i.e., symmetrically altered sensation in the distal lower extremities that improved proximally. Most subjects reported "negative" symptoms such as a sensation of "deadness" in the distal limbs, and a few reported positive symptoms at night as well. All PN subjects had signs consistent with PN as determined by the Michigan Diabetes Neuropathy Score (MDNS), ${ }^{8}$ which is a $0-46$ point scale. A higher score reflects more severe DPN, and it correlates well with more extensive neuropathy staging scales. The scale includes muscle stretch reflexes at the biceps, triceps, patella, and Achilles tendons; ability to perceive a pin, a $10 \mathrm{~g}$ monofilament, and $128 \mathrm{~Hz}$ tuning fork at the great toes; and strength of hand dorsal interossei, great toe extensors, and ankle dorsiflexors determined bilaterally. All PN subjects had MDNS scores of 10 or greater, which in previous work was found to be an appropriate cutoff for older persons. ${ }^{23}$ Lastly, all PN subjects had electrodiagnostic evidence of a diffuse $\mathrm{PN}$ as evidenced by abnormal sural sensory and peroneal motor nerve conduction studies. The abnormal responses were obtained bilaterally, as has been suggested in the definition of PN for the purposes of clinical research. ${ }^{7}$ Abnormal sural sensory nerve action potentials were defined as being absent, or with amplitudes of less than $6 \mu \mathrm{V}$ and/or latencies of greater than $4.2 \mathrm{~ms}$ stimulating $14 \mathrm{~cm}$ from the recording site posterior to the lateral malleolus. Peroneal compound muscle action potentials (CMAPs) were obtained by recording over the extensor digitorum brevis muscle and stimulating $9 \mathrm{~cm}$ proximally over the deep peroneal nerve and distal to the fibular head. Abnormal peroneal CMAPs were defined as absent or with amplitudes less than $2 \mathrm{mV}$, and/or latencies greater than $6.2 \mathrm{~ms}$, and/or conduction velocities less than $41.0 \mathrm{~m} / \mathrm{s}$.

Control subjects were matched to the PN subjects by, in order of priority, gender, age, height, and weight. Control subjects reported neither positive nor negative neurologic symptoms in the lower extremities. Control subjects also underwent the same physical examination as the PN subjects, and all had MDNS scores of less than 10. Therefore, the control subjects had neither signs nor symptoms of PN, thus the presence of $\mathrm{PN}$ in these subjects is highly unlikely. ${ }^{7}$

Experimental Apparatus. A servomotor-driven foot cradle system, which we have used in previous work, ${ }^{9}$ was created to evaluate ankle proprioceptive thresholds in the frontal plane (Fig. 1). An Aerotech servomotor (Model Number: 1000DC, Aerotech, Pittsburgh, Pennsylvania; continuous torque capability: 80 oz-in) was connected to the foot cradle via an aircraft cable and pulley system that produces rotation of the cradle system. To allow for finer control of the cradle, an additional 6000 line pulse encoder was installed on the rotational axis of the cradle. The depth of the cradle was matched to the height of the subtalar axis of ankle rotation so that the axes of the two were collinear, and a raised dummy plate was placed under the contralateral foot to bring the feet to the same level. The system was controlled by a custom LabView (National Instruments, Baltimore, Maryland) program on an IBM-compatible PC platform. The LabView program interfaced with a Mektronix MC-03 (Mektronics, Australia) motor controller board and National Instruments high-resolution 


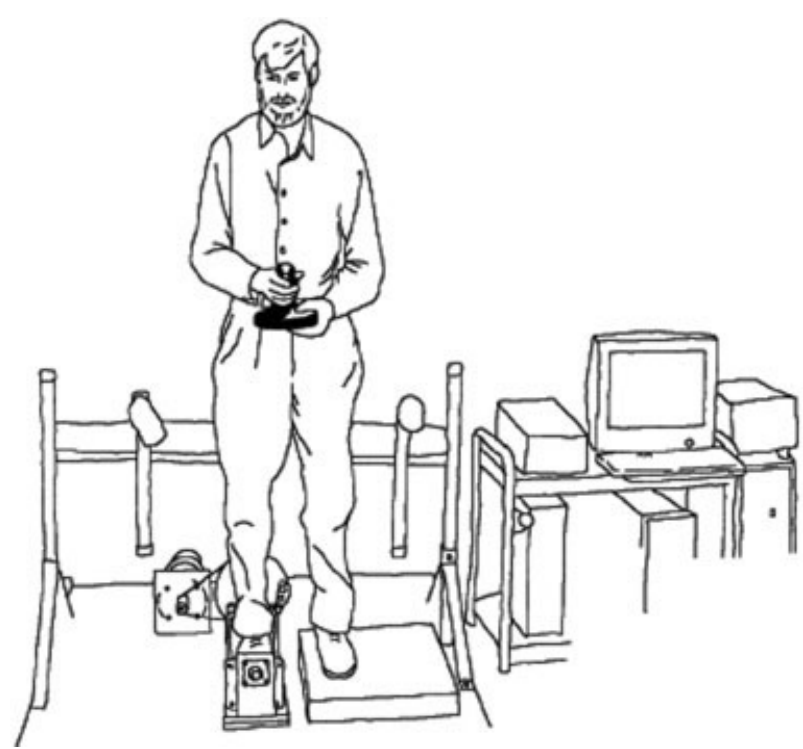

FIGURE 1. The experimental apparatus as described in the text.

multifunction I/O board (AT-MIO-16XE-50). A PCgame joystick was installed so the subject could provide feedback on the direction of any perceived ankle rotation to the computer. To ensure subject safety, infrared light beams were attached to each handrail to serve as emergency cutoff switches should the rails be touched. An additional emergency cutoff switch was available to the operator as well. Finally, limit switches and mechanical stops were provided that limited the range of ankle rotation to a maximum of $15^{\circ}$ of inversion or eversion beyond neutral ankle position.

Subject Protocols. Determining Proprioceptive Thresholds. The subject was asked to place his/her right foot inside the cradle, which was adjusted so the sub-talar joint axis was aligned as closely as possible with the rotational axis of the cradle. The heel and second metatarsal joint of the foot were aligned with the longitudinal axis of the cradle. The left foot was placed on a fixed plate at the same height as the surface of the cradle. The subject was instructed to place half the body weight on each foot and stand without assistance, looking forward while holding a joystick at the level of the umbilicus (Fig. 1). When the LabView program started to run, it provided an audible warning cue, after which the control program generated either a single ankle rotation (inversion or eversion) at $5 \%$ or no rotation (dummy trial). The subject then pressed the joystick handle in the direction of the perceived foot rotation. Further stimuli of randomized magnitude were then presented in a similar fashion using a staircase ap- proach. ${ }^{9}$ The program evaluated the proprioceptive threshold of the subject in real-time and adjusted the ankle rotation displacements by $0.1^{\circ}$ increments, between a minimum of 0.1 and a maximum of $1.0^{\circ}$, to fit the subject's proprioceptive ability. Based on our previous work, proprioceptive thresholds greater than $1.0^{\circ}$ in either inversion or eversion were not quantified so as to limit the length of the test protocol. A total of 100 trials were presented to each subject. The subject was instructed not to guess the direction of rotation, and 20 dummy no-motion trials were randomized into the 100 data trials to prevent guessing. During the dummy trials the motor was active to eliminate auditory clues to assist in discriminating between real and dummy trials.

Determining UPB Time. Subjects were instructed to maintain UPB for as long as possible. They were asked to not touch their lifted foot to the floor, or the stance limb, unless it was necessary to restore balance. The starting point for UPB was lift-off of the nonstance limb, and the endpoint was touching the nonstance limb to the floor or the stance limb, or shifting/sliding of the stance limb. A stopwatch was used to determine the elapsed time. Subjects stood with arms crossed in front of the chest during UPB testing. Given the absence of a laterality effect on $\mathrm{UPB},{ }^{2,10}$ the subjects performed three trials using the right foot followed by three trials using the left foot with rest given as required between each trial. An experienced physical therapist stood near the subject to act as a spotter in the event of an uncontrolled loss of balance.

Statistical Analyses. DeterminingProprioceptive Thresholds. Ankle proprioception was represented by an outcome measure, $\mathrm{TH}_{75}$, which was defined as the smallest rotational displacement of the ankle that a subject could identify with a $75 \%$ probability of success. ${ }^{31}$ To find $\mathrm{TH}_{75}$ the data, which were assumed to have the form of the Gaussian cumulative distribution function, were filtered using weighted threepoint averaging. A ceiling algorithm was then applied to transform the data into a monotonic function. Because many results showed step-wise increases in the probability graphs, with jumps in success rate from $0 \%$ to $100 \%$ for successive stimuli amplitudes, a continuous regression function (probit) was used to induce greater error. The intersection at $50 \%$ success rate $\left(\mathrm{TH}_{50}\right)$ was identified using linear interpolation between the two points just above and below $50 \%$. If two consecutive points shared identical probability, the next nearest point was used for the analysis. This point was assumed to be the mean of the Gaussian probability distribution 
function, and the slope at the point was assumed to be the standard deviation (SD) of that probability distribution function. The $\mathrm{TH}_{75}$ was calculated from this information using a custom MatLab program (MathWorks, Cambridge, Massachusetts) ${ }^{18}$ developed for that purpose. Microsoft Excel and SPSS (Chicago, Illinois) ${ }^{28}$ were used for statistical processing. Because the maximum rotational stimulus presented to the subjects was $1.0^{\circ}$, thresholds that were greater than this were deemed unreliable. Two PN subjects, who were found to have thresholds greater than $1.0^{\circ}$ were excluded from the linear regression and group comparison analyses described below.

Determining UPB Times. UPB time was calculated as the median value of the total (6) unipedal balance trials. The median value was chosen to minimize the ceiling effect related to the maximum UPB of $30 \mathrm{~s}$ and the small number of trials.

H1: Relationship between UPB and Ankle Proprioceptive Thresholds. Because the goal of the study was to identify the relationship between ankle proprioceptive thresholds and UPB, the latter of which involves bidirectional displacements, the $T H_{75}$ for eversion and inversion were summed. The relationship between the combined variable, $\mathrm{TH}_{75 \mathrm{Inv}+\mathrm{Ev}}$, and UPB time was explored using regression analysis. UPB time, with and without logarithmic transformation, was the outcome variable of interest. $\mathrm{TH}_{75 \text { Inv }+\mathrm{Ev}}$ and, secondarily, demographic variables (age, height, and weight) were explored as predictor variables. If more than one significant relationship with UPB time was identified, the independence of these variables was determined with multiple regression.

Although the PN and control subjects demonstrated significant differences in UPB time and proprioceptive thresholds, there was overlap between the two groups in both measures (Fig. 2). Furthermore, inspection of Figure 2 reveals that there are no extreme or "outlier" data points which might skew the regression equation. The data are therefore consistent with the subject makeup, which included healthy older persons, who are known to show mild signs of peripheral nerve dysfunction, and persons with mild to moderate PN. Therefore PN severity, UPB time, and proprioceptive threshold data all suggest that the subjects were on a continuum from absence of $\mathrm{PN}$ to moderate $\mathrm{PN}$, thus allowing regression analysis to be reasonably applied.

H2: Exploring Group Differences in UPB Time and Proprioceptive Thresholds. Group means were compared using two-sided, independent $t$-tests that assumed unequal variances, and the $P$ value was adjusted accordingly. Within subject means were

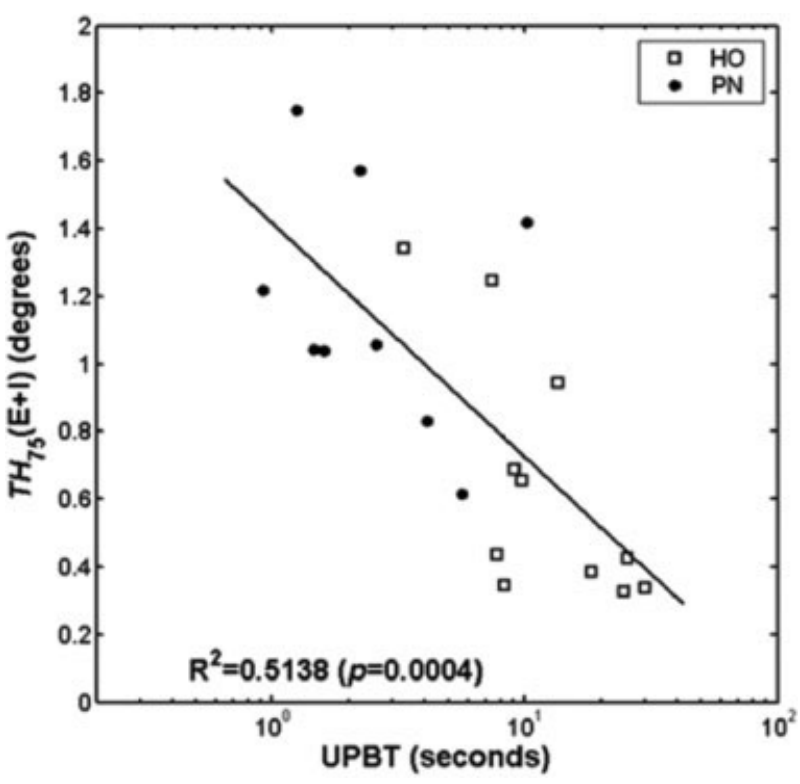

FIGURE 2. The relationship between combined ankle inversion and eversion proprioceptive thresholds and UPBT on a log-linear scale.

compared using paired two-sided $t$-tests. A $P$-value of 0.05 or less was considered significant.

\section{RESULTS}

Clinical and Demographic Data. Twenty-two subjects, $11 \mathrm{PN}$ and 11 controls, volunteered for the study. As would be anticipated from the matching protocol, there were no group differences in age, height, weight, and BMI (Table 1). The mean \pm SD MDNS scores for the PN and control subjects were $19.6 \pm$ 5.3 and $3.2 \pm 2.1$, and the range for these same scores were 11-26 and $0-7$, respectively. The PN subject scores are consistent with mild to moderate $\mathrm{PN}$, given that the maximum MDNS score (most severe) possible is 46 . This would be anticipated by the study criterion that prevented participation of PN subjects with ankle dorsiflexion strength less than 4 . The control subject scores indicate completely normal examinations, or minimal distal sensory dysfunction or Achilles reflex absence. Electrodiagnostic studies of the PN subjects demonstrated that none had recordable sural SNAPs. All PN subjects except one had recordable peroneal CMAPs, and the mean $\pm \mathrm{SD}$ response amplitudes, latencies and conduction velocities were $1.0 \pm 0.8 \mathrm{mV}, 5.7 \pm$ $0.7 \mathrm{~ms}$, and $37.1 \pm 3.6 \mathrm{~m} / \mathrm{s}$, respectively.

Primary Hypothesis: Relationship between $\mathbf{T H}_{\mathbf{7 5 I n v + E v}}$ and UPB Time. Two of the PN subjects demonstrated $\mathrm{TH}_{75 \mathrm{Inv}}$ and/or $\mathrm{TH}_{75 \mathrm{Ev}}>1.0^{\circ}$, and therefore 


\begin{tabular}{llll}
\hline & \multicolumn{2}{c}{ Table 1. Subject characteristics. } & \\
\hline & HO group & PN group & $P$-value* \\
\hline Number $(n)$ & 11 & 11 & $7 \mathrm{M}+4 \mathrm{~F}$ \\
Gender & $7 \mathrm{M}+4 \mathrm{~F}$ & $73.09 \pm 5.59$ & 0.7159 \\
Age $(\mathrm{yrs}$, mean $\pm \mathrm{SD})$ & $72.09 \pm 7.02$ & $1.71 \pm 0.12$ & 0.6917 \\
Height $(\mathrm{m})$ & $1.69 \pm 0.09$ & $88.78 \pm 17.15$ & 0.5514 \\
Weight $(\mathrm{kg})$ & $84.17 \pm 18.51$ & $30.16 \pm 4.08$ & 0.6459 \\
BMl $\left(\mathrm{kg} / \mathrm{m}^{2}\right)$ & $29.19 \pm 5.52$ & $19.6 \pm 5.3$ & $<0.0001$ \\
MDNS & $3.2 \pm 2.1$ & & \\
\hline
\end{tabular}

${ }^{*}$ Two PN subjects were excluded due to proprioceptive thresholds being $>1.0$ degree.

data from these subjects were excluded from the analysis. A significant inverse relationship (beta $=$ $-0.692 ; 95 \%$ confidence interval [CI] $=-1.025$, -0.359 ) between $\mathrm{TH}_{75 \mathrm{Inv}+\mathrm{Ev}}$ and the logarithmically transformed UPB time was identified, supporting the primary hypothesis (Fig. 2). This relationship demonstrated an $\mathrm{R}^{2}$ value slightly greater than 0.50 , suggesting that $\mathrm{TH}_{75 \mathrm{Inv}+\mathrm{Ev}}$ explains about one-half of the variance in clinical UPB time. There were no significant relationships identified between UPB time or $\mathrm{TH}_{75 \mathrm{Inv}+\mathrm{Ev}}$ and age, height, or weight.

Secondary Hypotheses: PN versus Control Group Comparisons. Proprioceptive Thresholds. Again, the two PN subjects with thresholds $>1.0^{\circ}$ were not included. The remaining $\mathrm{PN}$ subjects demonstrated proprioceptive thresholds, both inversion and eversion, that were significantly greater (less precise) than those of the control group. Those of the PN subjects were approximately twice those of the controls. These results support the secondary hypothesis regarding group differences in proprioceptive thresholds.

UPB Times. The PN group demonstrated UPB times that were significantly less than those of the control group. The PN group UPB times were less than one-fourth those of the controls (Table 2). The results support the secondary hypothesis regarding group differences in UPB time. Consistent with previous work, ${ }^{2}$ there was no side to side difference in UPB time $(P=0.6756$ and 0.7462 for the PN and control groups, respectively).

\section{DISCUSSION}

This study demonstrated that in older persons with and without diabetic PN frontal plane ankle proprioceptive thresholds demonstrate a negative relationship with clinical UPB time; i.e., as proprioceptive thresholds increase, or become worse, UPB time decreases. Moreover, the proprioceptive thresholds appear to explain approximately one-half of the variance in clinical UPB time. Although other work has identified increased ankle proprioceptive thresholds in older persons with $\mathrm{PN},{ }^{27,31}$ we are not aware of any study that has linked such thresholds with mobility function.

UPB time is thought to be the most challenging clinical balance task routinely given to patients. ${ }^{33}$ It is also a clinically important measure given its potent relationship to aging, ${ }^{2,21} \mathrm{PN},{ }^{13,24}$ current activity level in older persons, ${ }^{4}$ and falls in older persons with $^{22}$ and without PN. ${ }^{14,32}$ Figure 2 suggests that $\mathrm{TH}_{75 \mathrm{Inv}+\mathrm{Ev}}<1.0^{\circ}$ is necessary to reliably achieve $\geq 8-10$ s of UPS. Given other work that suggests that achieving between 5 and $10 \mathrm{~s}$ of UPB time reduces

\begin{tabular}{|c|c|c|c|c|}
\hline & Control & PN & PN/control & $P$-value \\
\hline \multicolumn{5}{|l|}{$\mathrm{TH}_{75}\left({ }^{\circ}\right)^{\star}$} \\
\hline Eversion & $0.43 \pm 0.26$ & $0.68 \pm 0.25$ & 1.58 & 0.0420 \\
\hline Inversion & $0.22 \pm 0.14$ & $0.49 \pm 0.21$ & 2.23 & 0.0052 \\
\hline Ev+lnv & $0.65 \pm 0.37$ & $1.17 \pm 0.36$ & 1.80 & 0.0055 \\
\hline \multicolumn{5}{|c|}{ Median UPBT (sec) } \\
\hline Right foot & $15.93 \pm 10.74$ & $3.31 \pm 2.95$ & 0.21 & 0.0022 \\
\hline Left foot & $14.68 \pm 10.71$ & $3.59 \pm 2.03$ & 0.24 & 0.0056 \\
\hline Right and left & $14.34 \pm 8.93$ & $3.39 \pm 2.72$ & 0.24 & 0.0017 \\
\hline
\end{tabular}

*Two PN subjects were excluded due to proprioceptive thresholds being $>1.0$ degree. 
fall risk in older persons with ${ }^{22}$ and without $\mathrm{PN},{ }^{32}$ the $1.0^{\circ}$ of $\mathrm{TH}_{75 \mathrm{Inv}+\mathrm{Ev}}$ is likely a clinically relevant threshold above which postural stability is significantly decreased.

Unmeasured factors likely determine the remainder of the variance in UPB time. Although age, height, and weight did not show significant relationships with UPB time in the subjects studied, neuropsychologic factors such as fear or confidence, and lifestyle variables such as routine level of physical activity, were not measured and likely played a role in the subjects' abilities to reliably stand on one foot. Similarly, unmeasured differences in vestibular, visual-perceptual, or musculoskeletal function also may have contributed to the unaccounted variance in UPB time. Ankle motor function, which is also influenced by $\mathrm{PN}$, is likely to have played a role in determining UPB time. In previous work we found that rate of ankle torque generation in the frontal plane explained over one-half of the variance in UPB time in a group of older women with diabetes, one-half of whom had PN. ${ }^{12}$ Determining whether afferent or efferent peripheral neurologic function is more important in determining UPB time, and the extent of collinearity between the two functions, requires the quantification of afferent and efferent ankle function within the same set of subjects. However, consideration of how these functions are used to maintain UPB allows some speculation on the matter.

For successful UPB the whole body center of mass, which drifts in the horizontal plane about $1 \mathrm{~m}$ above the support surface in a standing human, must be controlled and maintained directly above the stance foot. To do so requires rapid and precise information about the location of the center of pressure under the foot, as well as the rapid generation of torques at the ankle with which to manipulate it. Finer, or smaller, ankle proprioceptive thresholds would be expected to provide earlier information regarding the direction of movement of the lower leg relative to the foot than coarser, or larger, thresholds. Once leg motion is sensed, a rapid change in ankle torque is necessary to counteract the motion; e.g., when the lower leg drifts laterally, rapid ankle inversion is necessary to drive the center of pressure (and hence the ground reaction force) laterally to arrest the motion. Consistent with these concepts, healthy subjects with anesthetized foot soles do not increase center of pressure excursions as compared to the unanesthetized condition. They do show increased center of pressure velocities and plantar surface shear force, ${ }^{19}$ suggesting increased center of mass accelerations during the maintenance of balance. In other words, these subjects with impaired afferent, but intact efferent, function require more aggressive use of motor function to compensate for a delayed perception of body sway. Conversely, it would be anticipated that patients with highly precise afferent function would require less restorative ankle torque, because body sway would be reduced, this in turn would require less force to control. This model suggests, therefore, that afferent and efferent functions are independent and complementary influences on stability during UPB. Therefore, both are targets of similar import for potential intervention.

The hip also has an important role in maintaining frontal plane balance. Townsend ${ }^{29}$ and MacKinnon and Winter ${ }^{16}$ found that frontal plane balance was influenced most powerfully during gait by medial-lateral foot placement relative to the body center of mass. During unipedal stance the hip abductors primarily control the head, arms, trunk, and swing limb by preventing the lowering of these segments toward the unsupported limb. MacKinnon and Winter suggest that the subtalar and hip joints work in concert to control frontal plane balance during single limb stance and, furthermore, that "a communicative link exists between the state of the whole body balance system relative to the support surface and the musculature which controls the balance and posture of the largest proportion of body mass (the head, arms and trunk)."16 It seems likely that PN weakens this link and renders the hip musculature less able to respond rapidly and appropriately to perturbations. However, it is also possible that increasing $\mathrm{PN}$ is associated with weakness of the hip musculature, and the latter is responsible for the difficulty with UPB experienced by the PN subjects. This possibility seems less likely given the fact that PN is predominantly a distal process and would not be expected to directly influence hip strength.

The study also corroborated previous work that older persons with PN have increased ankle proprioceptive thresholds compared to control subjects. Simoneau et al. ${ }^{27}$ found that subjects with diabetic PN had increased proprioceptive thresholds in the sagittal plane. Although the magnitude of the difference in proprioceptive thresholds of the PN subjects compared to controls in Simoneau et al.'s work was similar to our study (about three times greater), the absolute values for the proprioceptive thresholds were dissimilar $\left(4.5 \pm 4.3^{\circ}\right.$ and $1.5 \pm 0.7^{\circ}$ for $\mathrm{PN}$ and control subjects in Simoneau et al.). This may be due to between-study differences in subject neuropathy severity, ankle plane of motion tested (sagittal plane vs. frontal), or testing technique. With regard to the last concern, Simoneau et al. used a slowly rotating 
$\left(0.25\right.$ or $\left.0.75^{\circ} / \mathrm{s}\right)$ platform that required the subjects to respond as soon as they perceived motion. This is in contrast with our technique, which presented discrete stimuli at a rotational speed of $5^{\circ} / \mathrm{s}$ and were increased or decreased depending on response accuracy. Van den Bosch et al. ${ }^{31}$ also reported increased frontal plane ankle proprioceptive thresholds in older persons with $\mathrm{PN}$, noting a PN-to-control subject to ratio of about $6: 1$. The thresholds were greater $\left(1.30 \pm 1.06\right.$ and $2.57 \pm 2.90^{\circ}$ for inversion and eversion, respectively) for van den Bosch et al., possibly due to subject differences, the slower rotation speed ( 2 vs. $5^{\circ} / \mathrm{s}$ ) of stimuli, or our exclusion of subjects with thresholds greater than $1.0^{\circ}$.

Our study also substantiated previous findings of a PN-related impairment in UPB. This finding was prominent in three other studies. ${ }^{1,13,24}$ The data, when considered in aggregate, suggest that UPB time greater than $10 \mathrm{~s}$ is rare in persons with large fiber PN, and when it is present, fall risk is relatively low. ${ }^{22}$

Determining proprioceptive thresholds is technically difficult, and the present techniques had disadvantages. One disadvantage is that the normal sway that occurs during quiet standing could, when in or out of phase with the direction of ankle rotation, reduce or augment the stimulus intensity. For example, when testing the right foot, an inverting stimulus would be attenuated by the coincidence of lateral sway to the right, but it would be accentuated by concurrent sway to the left. The alternative, testing performed in an open chain fashion with the foot and ankle nonweight bearing, avoids this potential source of error but at the cost of loss of construct validity due to the absence of plantar pressure sensation and reduced muscle spindle activation, both of which are normally present during the stance phase of gait. Proprioceptive testing was performed during bipedal rather than unipedal stance because of uncertainty in interpreting the results from the latter in previous work. In that work testing in unipedal stance required that the PN subjects be supported proximally by means of a thigh pad and upper extremity touch. It was unclear whether this extra support, or the increased loading of the stance foot and ankle, was responsible for the decreased (improved) proprioceptive thresholds found in that condition as compared to bipedal stance. ${ }^{31}$ In addition, the testing required sustained attention which may be affected by diabetes mellitus. ${ }^{17}$ However, other studies suggest that the influence of diabetes mellitus on cognitive function is uncertain ${ }^{6}$ and that there is no relationship between neuropsychologic testing and PN. ${ }^{15}$ Therefore, it seems unlikely that cognitive dysfunction completely explains the impairments in proprioception and UPB time, particularly given the work by Uciolli et al., ${ }^{30}$ which found postural stability in subjects with diabetic neuropathy to be quantitatively related to peripheral, but not central, nerve conduction study parameters.

In summary, frontal plane ankle proprioceptive thresholds predicted about one-half of the variance in UPB time in a group of older persons with and without PN. The findings suggest a link between $\mathrm{PN}$-associated loss of distal sensation and postural stability. Since prior work demonstrated a similarly strong relationship between ankle rate of torque generation and UPB time, ${ }^{12}$ PN-associated impairments in ankle frontal plane afferent and efferent function appear equally responsible for the inability of older persons with PN to reliably balance on one foot. They therefore provide distinct targets for intervention.

Supported by PHS grant 1P30 AG 08808 (to all). J.K.R. was supported by PHS grants K23 AG 00989-01 and RO1 AG 02656902. A portion of the material was presented, in abstract form, at the American Association of Neuromuscular and Electrodiagnostic Medicine meeting in Phoenix, Arizona on October 20, 2007.

\section{REFERENCES}

1. Ashton-Miller JA, Yeh MW, Richardson JK, Galloway T. A cane reduces loss of balance in patients with peripheral neuropathy: results from a challenging unipedal balance test. Arch Phys Med Rehabil 1996;77:446-452.

2. Bohannon RW, Larkin PA, Cook AC, Gear J, Singer J. Decrease in timed balance test scores with aging. Phys Ther 1984;64:1067-1070.

3. Boulton AJM, Vinik AT, Arezzo JC, et al. Diabetic neuropathies. A statement by the American Diabetes Association. Diabetes Care 2005;28:956-962.

4. Bulbulian R, Hargan ML. The effect of activity history and current activity on static and dynamic postural balance in older adults. Physiol Behav 200;70:319-325.

5. Cavanagh PR, Derr JA, Ulbrecht JS, Maser RE, Orchard TJ. Problems with gait and posture in neuropathic patients with insulin-dependent diabetes mellitus. Diabetes Med 1992;9: 469-474.

6. Coker LH, Shumaker SA. Type 2 diabetes mellitus and cognition: an understudied issue in women's health. J Psychosom Res 2003;54:129-139.

7. England JD, Gronseth GS, Franklin G, Miller RG, Asbury AK, Carter GT, et al. Distal symmetrical polyneuropathy: definition for clinical research. Muscle Nerve 2005;31:113-123.

8. Feldman EL, Stevens MJ, Thomas PK, Brown MB, Canal N, Greene DA. A practical two-step quantitative clinical and electrophysiological assessment for the diagnosis and staging of diabetic neuropathy. Diabetes Care 1994;17:1281-1289.

9. Gilsing MG, Van den Bosch CG, Lee SG, Ashton-Miller JA, Alexander NB, Schultz AB, et al. Association of age with the threshold for detecting ankle inversion and eversion in upright stance. Age Ageing 1995;24:58-66.

10. Goldie PA, Evans OM, Bach TM. Steadiness in one-legged stance: development of a reliable force-platform testing procedure. Arch Phys Med Rehabil 1992;73:348-354. 
11. Greenspan SL, Meyers ER, Maitland LA, Resnick NJ, Hayes WC. Fall severity and bone mineral density as risk factors for hip fracture in ambulatory elderly. JAMA 1994;271:128-133.

12. Gutierrez MS, Helber MB, Dealva D, Ashton-Miller, Richardson JK. Mild diabetic neuropathy affects ankle motor function. Clin Biomech 2001;16:522-528.

13. Hurvitz EA, Richardson JK, Werner RA. Unipedal stance testing in the assessment of peripheral neuropathy. Arch Phys Med Rehabil 2001;82:198-204.

14. Hurvitz EA, Richardson JK, Werner RA, Ruhl A, Dixon M. Unipedal stance testing as an indicator of fall risk among older outpatients. Arch Phys Med Rehabil 2000;81:587-591.

15. Lawson JS, Erdahl DLW, Monga TN, Bird CE, Donald MW, Surridge DHC, et al. Neuropsychological function in diabetic patients with neuropathy. Br J Psychiatry 1984;145:263-268.

16. MacKinnon CD, Winter DA. Control of whole body balance in the frontal plane during human walking. J Biomech 1993;26: 633-644.

17. Manschot SM, Brands AM, van der Grond J, Kessels RP, Algra A, Kappelle LJ, et al. Brain magnetic resonance imaging correlates of impaired cognition in patients with type 2 diabetes. Diabetes 2006;55:1106-1113.

18. MATLAB program, The MathWorks, Cambridge, MA

19. Meyer PF, Oddsson LIE, DeLuca CJ. The role of plantar cutaneous sensation in unperturbed stance. Exp Brain Res 2004;156:505-512.

20. Mold JW, Vesely SK, Keyl BA, Schenk JB, Roberts M. The prevalence, predictors, and consequences of peripheral sensory neuropathy in older patients. J Am Board Fam Pract 2004;14:309-318.

21. Potvin AR, Syndulko K, Tourtellotte K, Lemmon WW, Potvin $\mathrm{JH}$. Human neurologic function and the aging process. J Am Geriatr Soc 1980;28:1-9.

22. Richardson JK. Factors associated with falls in older patients with diffuse polyneuropathy. J Am Geriatr Soc 2002;50:17671773 .
23. Richardson JK. The clinical identification of peripheral neuropathy among older persons. Arch Phys Med Rehabil 2002; 83:1553-1558.

24. Richardson JK, Ashton-Miller JA, Lee SG, Jacob K. Moderate peripheral neuropathy impairs weight transfer and unipedal balance in the elderly. Arch Phys Med Rehabil 1996;77:11521156.

25. Richardson JK, Ching C, Hurvitz EA. The relationship between electromyographically documented peripheral neuropathy and falls. J Am Geriatr Soc 1992;40:1008-1012.

26. Richardson JK, Hurvitz EA. Peripheral neuropathy: a true risk factor for falls. J Gerontol Med Sci 1995;50A:M211-M215.

27. Simoneau GG, Derr JA, Ulbrecht JS, Becker MB, Cavanagh PR. Diabetic sensory neuropathy effect on ankle joint movement perception. Arch Phys Med Rehabil 1996;77:453-460.

28. SPSS program, SPSS Inc., Chicago, IL.

29. Townsend MA. Dynamics and coordination of torso motions inhuman locomotion. J Biomech 1981;14:727-738.

30. Uccioli L, Gicomini PG, Pasqualetti P, DiGirolamo S, Ferrigno $\mathrm{P}$, Monticone $\mathrm{G}$, et al. Contribution of central neuropathy to postural instability in IDDM patients with peripheral neuropathy. Diabetes Care 1997;20:929-934.

31. Van den Bosch C, Gilsing MG, Lee SG, Richardson JK, Ashton-Miller JA. Peripheral neuropathy effect on ankle inversion and eversion detection thresholds. Arch Phys Med Rehabil 1995;76:850-856.

32. Vellas BJ, Wayne SJ, Romero L, Baumgartner RN, Rubenstein LZ, Garry PJ. One-leg balance is an important predictor of injurious falls in older persons. J Am Geriatr Soc 1997;45:735738.

33. Wang CY, Hsieh CL, Olson SL, Wang CH, Sheu CF, Liang CC. Psychometric properties of the Berg Balance Scale in a community-dwelling elderly resident population in Taiwan. J Formosan Med Assoc 2006;105:992-1000. 